\title{
Effect of Parapheromones on the Capture of Fruit Flies (Diptera: Tephritidae) in Burundi
}

\author{
Liévin Ndayizeye ${ }^{1,2}$, Benoît Nzigidahera ${ }^{1}$ and Charmaine Dawn Theron ${ }^{3}$ \\ 1. Office Burundais pour la Protection de l'Environnement (OBPE), B.P. 2757, Bujumbura, Burundi \\ 2. Université du Burundi, Institut de Pédagogie Appliquée, B.P. 5223, Bujumbura, Burundi \\ 3. Department of Zoology and Entomology, University of Pretoria, Pretoria 0012, South Africa
}

\begin{abstract}
This study aimed to make an inventory of fruit fly species in Burundi, by use of parapheromones. The trapping by parapheromones is a method used to capture fruit fly species. Four types of parapheromones were used, namely, methyl eugenol, cuelure, terpinyl acetate and trimedlure. In total, 70,115 individuals were captured, of which, 68,728 individuals (or 98.02\%) were fruit flies, while 1,387 individuals (1.98\%) were non-fruit flies. The majority of Tephritidae has been captured by methyl eugenol with 62,538 individuals $(90.97 \%)$, followed by cuelure with 3,621 individuals (5.27\%). Terpinyl acetate and trimedlure have captured 1,900 and 669 individuals, respectively. The effectiveness of parapheromones has varied from one locality to another. In fact, methyl eugenol showed efficiency in the city of Bujumbura, Rumonge, Kigwena and Cibitoke located at an altitude between $780 \mathrm{~m}$ and $887 \mathrm{~m}$; cuelure in Bubanza and Kayanza at an altitude between 1,101 m and 2,219 m; trimedlure and terpinyl acetate at Mwaro at an altitude of 1,640 m. From the point of view of abundance of species caught, Bactrocera dorsalis dominates with 62,349 individuals $(90.72 \%)$, and it is in the Bujumbura city that this species has been captured in abundance.
\end{abstract}

Key words: Abundance, distribution, effectiveness of parapheromones, localities, species.

\section{Introduction}

Fruit production provides a source of income and employment to many farmers [1]. Unfortunately, infestation by fruit flies is a major constraint to fruit production [2]. Tephritid fruit flies (Diptera: Tephritidae) cause devastating direct losses to many fresh fruits and vegetables [3].

Due to some species of Tephritidae, quarantine restrictions are imposed to prevent the transfer of foreign species from one region to another $[4,5]$. Without control, direct damage has been reported from $30 \%$ to $80 \%$, depending on the fruit variety, location and fruit season [6]. The damage caused by fruit flies is a major impediment in African countries. For example, in Kenya [7], as well as in Tanzania [8, 9], the fruit flies cause heavy losses to producers of mangoes.

Corresponding author: Liévin Ndayizeye, aggregate license $(\mathrm{bac}+5)$, research field: fruit flies.
In Burundi, the fruit production industry has grown markedly since 2005 with an increase in export. Many local businesses of transformation have been implanted. The produced fruits of economic significance are mandarins, oranges, mangoes, bananas, avocados, passion fruit, etc., which are sold on the local markets and exported to neighboring countries $[10,11]$. The agricultural industry has been experiencing losses due to spoilage and fruits which fall extensively prior to maturity as well as deformed fruits which are not appreciated on the market. Little information is available on the fruit flies of Burundi. Seeing the magnitude of damage, the identification of the major pests is required to develop an effective control strategy [12]. The identification of species is possible with parapheromones $[13,14]$ and for identifying adults that emerge from fruits [15]. In Burundi, research into the fruit fly diversity started in 2009 , in the framework of a research project on the fruit flies of natural and agricultural areas of Burundi. 
This project was initiated by the Burundi Environment Protection Authority (OBPE) with support from the Royal Museum for Central Africa (RMCA) in Tervuren, Belgium.

Thus, from 2009 up to 2014, studies using trapping by parapheromones, namely, methyl eugenol, cuelure, trimedlure and terpinyl acetate, have been carried out for the purpose of making an inventory of fruit flies of Burundi and testing the effectiveness of parapheromones in the capture of species in natural environments, agricultural and urban areas.

This paper presented a current state on the prevalence of fruit flies in Burundi, their abundance in the localities inspected, as well as the efficacy of parapheromones used.

\section{Methodology}

\subsection{Field Sites}

Research was conducted in 12 sampling sites across seven localities: three in Bujumbura, two in Bubanza, one in Rumonge, one in Kigwena, one in Cibitoke, one in Mwaro and three in Kayanza (Fig. 1). Field sites in a range of altitude from $780 \mathrm{~m}$ to $2,219 \mathrm{~m}$ (Table 1) were grouped into high (1,900-2,600 m), medium (1,400-1,700 m) and low altitude (750-1,300 m).

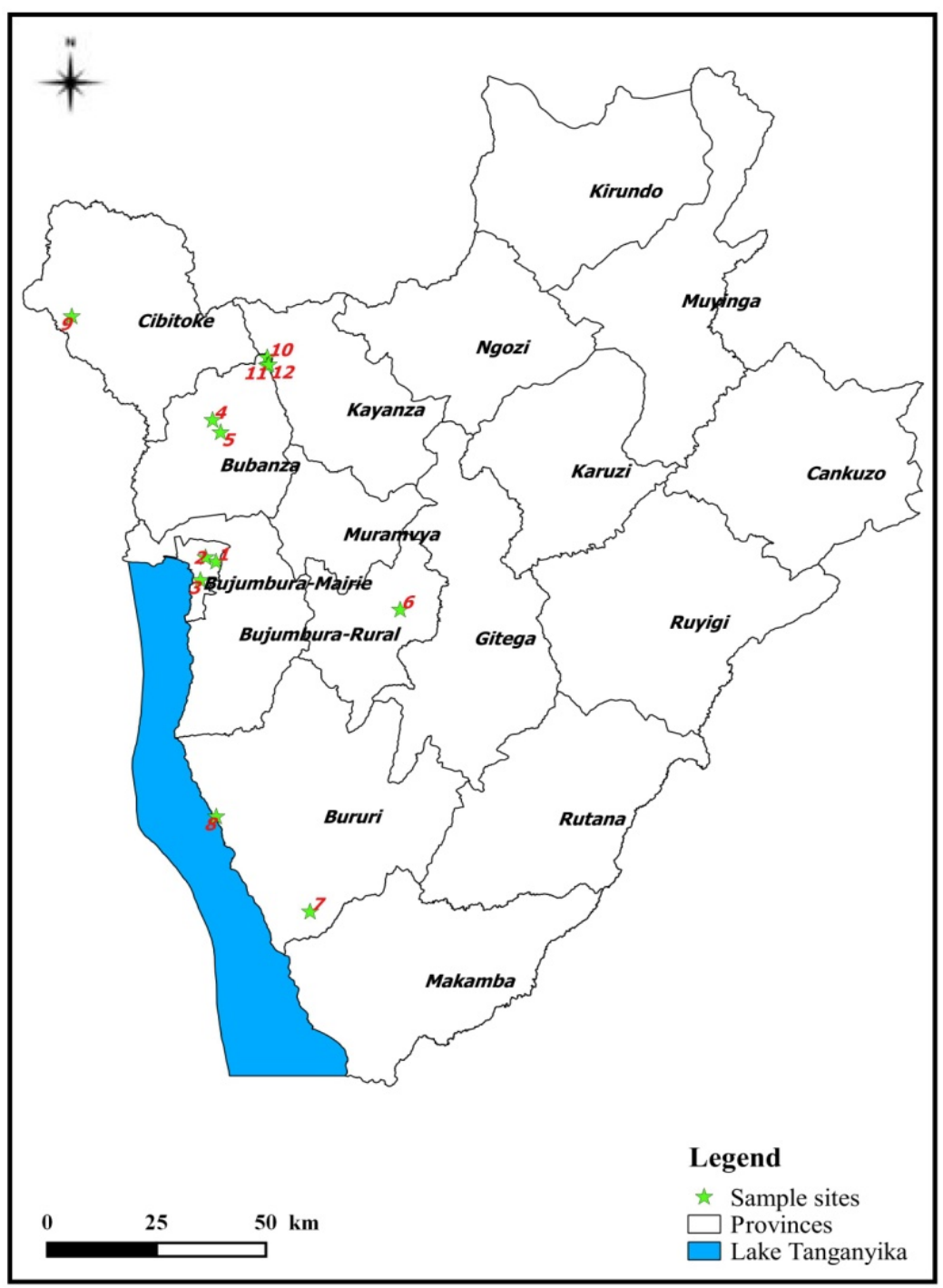

Fig. 1 Map representing different study sites across Burundi where various baited fruit fly traps were deployed for the period from November 2009 up to April 2014.

High altitudes: sites 10,11 and 12; medium altitude: site 6; low altitudes: sites 1, 2, 3, 4, 5, 7, 8 and 9. 
Table 1 Study sites in Burundi where Tephritidae-specific parapheromone traps were deployed (2009-2014).

\begin{tabular}{|c|c|c|c|c|c|}
\hline Level of altitudes & Localities & Sites & Coordinates & Altitudes & Habitats \\
\hline \multirow{8}{*}{ Low altitude } & \multirow{3}{*}{ Bujumbura } & Site 1 & $3^{\circ} 21^{\prime} 16^{\prime \prime} \mathrm{S}$ and $29^{\circ} 23^{\prime} 48^{\prime \prime} \mathrm{E}$ & $887 \mathrm{~m}$ & Green spaces, orchard \\
\hline & & Site 2 & $3^{\circ} 20^{\prime} 46^{\prime \prime} \mathrm{S}$ and $29^{\circ} 22^{\prime} 27^{\prime \prime} \mathrm{E}$ & $819 \mathrm{~m}$ & Farmfield \\
\hline & & Site 3 & $3^{\circ} 23^{\prime} 40^{\prime \prime} \mathrm{S}$ and $29^{\circ} 21^{\prime} 49^{\prime \prime} \mathrm{E}$ & $820 \mathrm{~m}$ & Orchard \\
\hline & Kigwena & Site 7 & $4^{\circ} 5^{\prime} 51^{\prime \prime} \mathrm{S}$ and $29^{\circ} 35^{\prime} 20^{\prime \prime} \mathrm{E}$ & $790 \mathrm{~m}$ & Forest, farmfield \\
\hline & Rumonge & Site 8 & $3^{\circ} 53^{\prime} 46^{\prime \prime} \mathrm{S}$ and $29^{\circ} 23^{\prime} 46^{\prime \prime} \mathrm{E}$ & $780 \mathrm{~m}$ & Farmfield \\
\hline & Cibitoke & Site 9 & $2^{\circ} 50^{\prime} 6^{\prime \prime} \mathrm{S}$ and $29^{\circ} 5^{\prime} 56^{\prime \prime} \mathrm{E}$ & $790 \mathrm{~m}$ & Orchard \\
\hline & \multirow{2}{*}{ Bubanza } & Site 4 & $3^{\circ} 3^{\prime} 17^{\prime \prime} \mathrm{S}$ and $29^{\circ} 23^{\prime} 19^{\prime \prime} \mathrm{E}$ & $1,248 \mathrm{~m}$ & Orchard \\
\hline & & Site 5 & $3^{\circ} 4^{\prime} 51^{\prime \prime} \mathrm{S}$ and $29^{\circ} 24^{\prime} 18^{\prime \prime} \mathrm{E}$ & $1,101 \mathrm{~m}$ & Farmfield \\
\hline Medium altitude & Mwaro & Site 6 & $3^{\circ} 27^{\prime} 25^{\prime \prime} \mathrm{S}$ and $29^{\circ} 46^{\prime} 26^{\prime \prime} \mathrm{E}$ & $1,640 \mathrm{~m}$ & Orchard \\
\hline \multirow{3}{*}{ High altitude } & \multirow{3}{*}{ Kayanza } & Site 10 & $2^{\circ} 55^{\prime} 18^{\prime \prime} \mathrm{S}$ and $29^{\circ} 30^{\prime} 4^{\prime \prime} \mathrm{E}$ & $2,219 \mathrm{~m}$ & Forest \\
\hline & & Site 11 & $2^{\circ} 56^{\prime} 21^{\prime \prime} \mathrm{S}$ and $29^{\circ} 30^{\prime} 1^{\prime \prime} \mathrm{E}$ & $2,212 \mathrm{~m}$ & Farmfield \\
\hline & & Site 12 & $2^{\circ} 56^{\prime} 14^{\prime \prime} \mathrm{S}$ and $29^{\circ} 30^{\prime} 13^{\prime \prime} \mathrm{E}$ & $2,172 \mathrm{~m}$ & Farmfield \\
\hline
\end{tabular}

The sites $1,2,3,4,5,7,8$ and 9 were low altitudes, the site 6 was medium altitude, and the sites 10, 11 and 12 were high altitudes. Field sites in Kayanza and Kigwena consisted of natural vegetation and agricultural ecosystems, while the remaining field sites were dominated by agricultural ecosystem. The field sites were sampled over a period of six months from November 2009 to April 2014.

\subsection{Collection of Fruit Flies}

Four types of parapheromones were used, namely, methyl eugenol, cuelure, terpinyl acetate and trimedlure from International Pheromone Systems (IPS), Wallasey, Wirral, UK. Lures were placed in Tephri-traps (Sorygar, Madrid, Espagne) with an insecticidal pad (dichlorvos 20\%) (IPS, Wallasey, Wirral, UK). The numbers of trees varied from 500 up to 2,200 for the orchards inspected. The distance between trees was roughly $6 \mathrm{~m}$ and the traps were suspended on branches at $1.30 \mathrm{~m}$ from the ground. The traps were suspended with the aid of a twine coated with strong grease to prevent predatory action of ants. Traps were emptied every two weeks and flies collected were placed in sample jars containing 70\% ethyl alcohol and labeled according to sample site and date. The type of tree and lure were also recorded for each sample. Lures and insecticide were replaced every six weeks based on efficacy periods according to Ekesi and Billah [16]. Fruit fly collection was done from November 2009 to April 2014. The co-ordinates of each field site were taken at the position of each Tephri-trap.

\subsection{Identification of Species of Fruit Flies}

Samples were first sorted on the basis of morphospecies, and then identification was done using published keys of Ekesi and Billah [16]. A voucher specimen was sent to the RMCA in Tervuren, Belgium to confirm the identification and preservation. The identification and confirmation of identified samples were done by Dr. Marc De Meyer.

\subsection{Data Analysis}

The index of Sorensen was used to compare the surveyed localities according to their faunistic richness. The index of Sorensen reveals the localities where species can fit well. Apart from this, it can show which species are particular to a given locality or area. Most of the time, these species are found in one locality abundantly.

The value of Sorensen similarity index $(K)$ [17] is obtained by the following Eq. (1):

$$
K=\frac{2 a}{2 a+b+c} \times 100
$$

where, $a$ is the number of species common to the two ecosystems, $b$ and $c$ are the numbers of species absent in one of the two ecosystems, but present in the other. 


\section{Results}

\subsection{Numbers of Flies Captured}

During the research period (November 2009-March 2014), total 70,115 individuals were collected, of which 68,728 (98.02\%) were Tephritidae fruit flies and $1,387(1.98 \%)$ were non-tephritid fruit flies and Hymenoptera. The majority of Tephritidae were captured by methyl eugenol (90.97\%), followed by cuelure (5.27\%). Terpinyl acetate and trimedlure accounted for 1,900 and 669 individuals, respectively (Table 2). Methyl eugenol has shown an attractive power of $99.39 \%$, followed by terpinyl acetate with $94.81 \%$. Trimedlure and cuelure have an attractive power of $85.66 \%$ and $82.09 \%$, respectively.

Non-tephritids (Diptera) and Hymenoptera represented $1.96 \%(1,373)$ and $0.02 \%$ (14) of trap captures, respectively. Diptera were found in the traps containing the four parapheromones, whereas the Hymenoptera have been found in the traps containing cuelure and methyl eugenol only. It should be noted that a lot of non-fruit flies were found in the traps containing cuelure, followed by methyl eugenol.

\subsection{Tephritidae Captured by Parapheromone in Different Sites}

There was a difference in response to parapheromones at the site level.

\subsubsection{Capture by Methyl Eugenol}

Bactrocera dorsalis Hendel was highly captured at low altitude sites (sites 1, 2, 3, 4, 5, 7 and 9). By comparison, B. dorsalis showed lower captures at middle and high altitude sites (sites 6, 10, 11 and 12).
The species of Ptiloniola edwardsi was captured extensively at site 10 , but not in sites 11 and 12 . This species could be weakly attracted to methyl eugenol. There was no dominant species captured at the medium altitude (site 6) (Table 3 ).

\subsubsection{Capture by Cuelure}

For cuelure, species Dacus punctatifrons Karsh, D. bivittatus Bigot, Zeugodacus cucurbitae Coquillet and Ptiloniola edwardsi were captured. D. punctatifrons was most abundant in sites 4 and 6 , and lower prevalence at sites 2, 3, 7 and 8. D. bivittatus was most abundant at site 5 , with lower prevalence at sites 1, 2, 3, 5 and 8. Z. cucurbitae was most abundant at site 2 and lower prevalence at sites 7, 8 and 9. $P$. edwardsi has been captured at low numbers only at site 10 . This species has also been captured by methyl eugenol.

\subsubsection{Capture by Terpinyl Acetate}

Considering terpinyl acetate, Ceratitis cosyra Walker and C. fasciventris Bezzi were most abundant. C. cosyra was abundant at site 1 , moderately abundant at site 2 and less abundant at site 3. Also, it was abundant at site 6 (medium altitude), but absent at sites 4 and 5 (low altitude), sites 10, 11 and 12 (high altitude).

\subsubsection{Capture by Trimedlure}

Trimedlure captured C. fasciventris and C. capitata Wiedemann. C. capitata was present at site 4 and absent at site 5. C. capitata was most abundant at site 9. C. fasciventris was only detected at site 6 .

In total, 24 species of fruit flies were captured. Methyl eugenol captured 14 different species with $B$. dorsalis the most dominant (99.60\%). Thirteen species

Table 2 Individuals captured by parapheromones across seven localities in Burundi.

\begin{tabular}{lllllll}
\hline Parapheromones & Cuelure & Methyl eugenol & Terpinyl acetate & Trimedlure & Total & $\%$ \\
\hline Tephritidae & 3,621 & 62,538 & 1,900 & 669 & 68,728 & 98.02 \\
\hline Non-tephritidae & & & & & & \\
\hline Diptera & 788 & 369 & 104 & 112 & 1,373 & 1.96 \\
Hymenoptera & 2 & 12 & 0 & 0 & 14 & 0.02 \\
Subtotal & 790 & 381 & 104 & 112 & 1,387 & 1.98 \\
\hline Total & 4,411 & 62,919 & 2,004 & 781 & 70,115 & 100 \\
Attractive power in \% & 82.09 & 99.39 & 94.81 & 85.66 & 98.02 & \\
\hline
\end{tabular}


Table 3 Variation of catches by parapheromones in different sites.

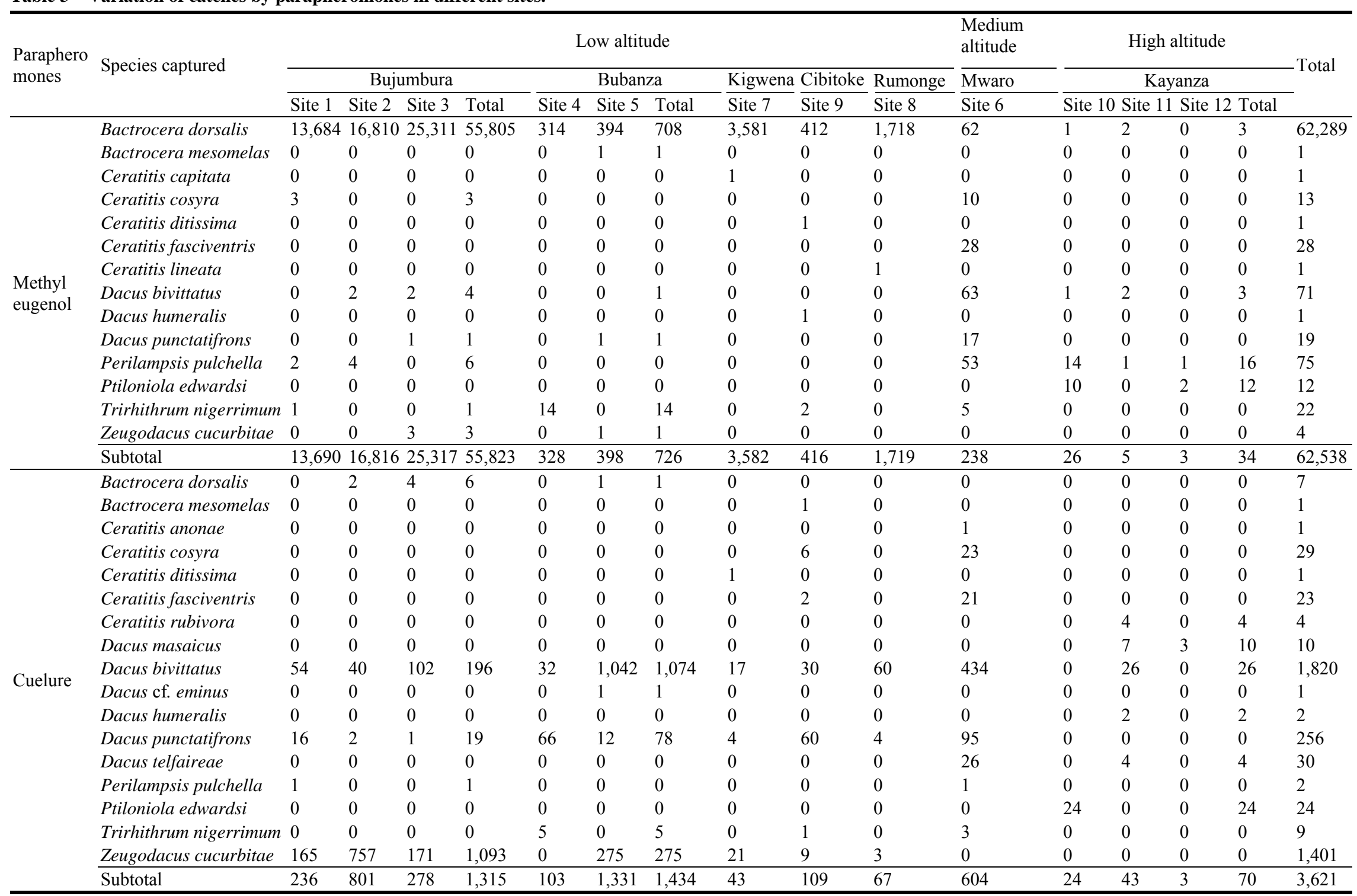


(Table 3 continued)

\begin{tabular}{|c|c|c|c|c|c|c|c|c|c|c|c|c|c|c|c|c|c|}
\hline \multirow{3}{*}{$\begin{array}{l}\text { Paraphero } \\
\text { mones }\end{array}$} & \multirow{3}{*}{ Species captured } & \multicolumn{10}{|c|}{ Low altitude } & \multirow{3}{*}{$\begin{array}{l}\begin{array}{l}\text { Medium } \\
\text { altitude }\end{array} \\
\text { Mwaro } \\
\text { Site } 6\end{array}$} & \multirow{2}{*}{\multicolumn{4}{|c|}{$\frac{\text { High altitude }}{\text { Kayanza }}$}} & \multirow{3}{*}{ Total } \\
\hline & & \multicolumn{4}{|c|}{ Bujumbura } & \multicolumn{3}{|c|}{ Bubanza } & \multirow{2}{*}{$\frac{\text { Kigwena }}{\text { Site } 7}$} & \multirow{2}{*}{$\frac{\text { Cibitoke }}{\text { Site } 9}$} & \multirow{2}{*}{$\begin{array}{l}\text { Rumonge } \\
\text { Site } 8\end{array}$} & & & & & & \\
\hline & & Site 1 & Site 2 & Site 3 & Total & Site 4 & Site 5 & Total & & & & & & $\mathrm{Si}$ & Site & Total & \\
\hline \multirow{14}{*}{$\begin{array}{l}\text { Terpinyl } \\
\text { acetate }\end{array}$} & Bactrocera dorsalis & 31 & 5 & 2 & 38 & 0 & 0 & 0 & 6 & 1 & 1 & 2 & 0 & 0 & 0 & 0 & 48 \\
\hline & $\begin{array}{l}\text { Bistrispinaria } \\
\text { magniceps }\end{array}$ & 0 & 0 & 0 & 0 & 1 & 0 & 1 & 0 & 0 & 0 & 0 & 0 & 0 & 0 & 0 & 1 \\
\hline & Ceratitis pallidula & 0 & 0 & 0 & 0 & 0 & 0 & 0 & 0 & 0 & 17 & 0 & 0 & 0 & 0 & 0 & 17 \\
\hline & Ceratitis anonae & 0 & 0 & 0 & 0 & 0 & 1 & 1 & 0 & 0 & 1 & 6 & 0 & 0 & 0 & 1 & 8 \\
\hline & Ceratitis capitata & 0 & 0 & 1 & 1 & 4 & 0 & 4 & 0 & 9 & 0 & 0 & 0 & 0 & 0 & 0 & 14 \\
\hline & Ceratitis cosyra & 608 & 195 & 48 & 851 & 0 & 0 & 0 & 29 & 23 & 29 & 135 & 0 & 0 & 0 & 0 & 1,038 \\
\hline & Ceratitis fasciventris & 0 & 0 & 1 & 1 & 10 & 3 & 13 & 0 & 1 & 0 & 632 & 0 & 0 & 0 & 0 & 647 \\
\hline & Dacus bivittatus & 0 & 0 & 0 & 0 & 0 & 0 & 0 & 0 & 33 & 0 & 39 & 0 & 2 & 0 & 2 & 74 \\
\hline & Dacus punctatifrons & 0 & 0 & 0 & 0 & 0 & 0 & 0 & 0 & 11 & 0 & 1 & 0 & 0 & 0 & 0 & 12 \\
\hline & Dacus telfaireae & 0 & 0 & 0 & 0 & 0 & 0 & 0 & 0 & 0 & 0 & 5 & 0 & 0 & 0 & 0 & 5 \\
\hline & Ptiloniola edwardsi & 0 & 0 & 0 & 0 & 0 & 0 & 0 & 0 & 0 & 0 & 0 & 0 & 1 & 0 & 1 & 1 \\
\hline & Trirhithrum nigerrimum & 0 & 0 & 0 & 0 & 3 & 0 & 3 & 0 & 0 & 0 & 12 & 0 & 0 & 0 & 0 & 16 \\
\hline & Zeugodacus cucurbitae & 0 & 0 & 0 & 0 & 0 & 1 & 1 & 0 & 18 & 0 & 0 & 0 & 0 & 0 & 0 & 19 \\
\hline & Subtotal & 639 & 200 & 52 & 891 & 18 & 5 & 23 & 35 & 96 & 48 & 832 & 0 & 3 & 0 & 4 & 1,900 \\
\hline \multirow{13}{*}{ Trimedlure } & Bactrocera dorsalis & 5 & 0 & 0 & 5 & 0 & 0 & 0 & 0 & 0 & 0 & 0 & 0 & 0 & 0 & 0 & 5 \\
\hline & Bactrocera mesomelas & 0 & 0 & 0 & 0 & 0 & 0 & 0 & 0 & 1 & 0 & 0 & 0 & 0 & 0 & 0 & 1 \\
\hline & Ceratitis anonae & 0 & 0 & 1 & 1 & 0 & 0 & 0 & 0 & 0 & 0 & 0 & 0 & 0 & 0 & 0 & 1 \\
\hline & Ceratitis capitata & 14 & 0 & 2 & 16 & 42 & 1 & 43 & 0 & 53 & 4 & 0 & 0 & 0 & 2 & 2 & 118 \\
\hline & Ceratitis cosyra & 0 & 0 & 0 & 0 & 0 & 0 & 0 & 0 & 0 & 0 & 9 & 0 & 0 & 0 & 0 & 9 \\
\hline & Ceratitis fasciventris & 0 & 0 & 3 & 3 & 0 & 0 & 0 & 0 & 1 & 0 & 515 & 0 & 0 & 0 & 0 & 519 \\
\hline & Ceratitis punctata & 0 & 0 & 0 & 0 & 0 & 0 & 0 & 1 & 0 & 1 & 0 & 0 & 0 & 0 & 0 & 2 \\
\hline & Ceratitis rubivora & 0 & 0 & 0 & 0 & 0 & 0 & 0 & 0 & 0 & 0 & 0 & 0 & 0 & 6 & 6 & 6 \\
\hline & Dacus bivittatus & 0 & 0 & 0 & 0 & 0 & 0 & 0 & 0 & 0 & 0 & 1 & 0 & 1 & 0 & 1 & 2 \\
\hline & Ptiloniola edwardsi & 0 & 0 & 0 & 0 & 0 & 0 & 0 & 0 & 0 & 0 & 0 & 1 & 0 & 0 & 1 & 1 \\
\hline & Trirhithrum coffeae & 0 & 0 & 0 & 0 & 0 & 0 & 0 & 0 & 0 & 0 & 0 & 0 & 0 & 2 & 2 & 2 \\
\hline & Trirhithrum nigerrimum & 0 & 0 & 0 & 0 & 3 & 0 & 3 & 0 & 0 & 0 & 1 & 0 & 0 & 0 & 0 & 3 \\
\hline & Subtotal & 19 & 0 & 6 & 25 & 45 & 1 & 46 & 1 & 55 & 5 & 526 & 1 & 1 & 10 & 12 & 669 \\
\hline
\end{tabular}


were captured by terpinyl acetate with two dominant species, namely, C. cosyra $(54.63 \%)$ and $C$. fasciventris $(34.05 \%)$. Cuelure captured 17 species, including two dominant species: D. bivitattus $(50.26 \%)$ and Z. cucurbitae (38.69\%). Trimedlure has captured 12 species dominated by $C$. fasciventris at $77.58 \%$. The remaining species were present in minor amounts (Table 4).

Generally speaking, for all species caught by parapheromones, $B$. dorsalis was the dominant species at a rate of $90.72 \%$.

\subsection{Distribution of Species by Localities}

Of 24 species captured, the localities of Bubanza and Cibitoke had the highest diversity of species (11 species), followed by Kayanza and the city of Bujumbura with 10 species. The locality of Rumonge had the lowest diversity with six species. Of all species identified, $B$. dorsalis was present at all the localities. It can be qualified as a widespread species. Nevertheless, its presence was the minimal at Mwaro with $2.91 \%$ and Kayanza with $2.52 \%$. An average rate of presence was also seen in Bubanza with a rate of $31.79 \%$. B. dorsalis was most abundant in Cibitoke, Kigwena, Rumonge and the city of Bujumbura. The highest rate of presence was at Kigwena with $98.38 \%$ and Cibitoke with $61.09 \%$ (Table 5).

D. bivittattus was moderately abundant at Bubanza (48.21\%), Kayanza (26.89\%) and Mwaro (24.41\%). These three localities are located in high altitude,

Table 4 Abundance of species by parapheromones.

\begin{tabular}{|c|c|c|c|c|c|c|c|c|c|c|}
\hline \multirow{2}{*}{ Species } & \multicolumn{8}{|c|}{ Abundance of species } & \multirow{2}{*}{ - Total } & \multirow{2}{*}{$\%$} \\
\hline & CUE & $\%$ & ME & $\%$ & TA & $\%$ & TRI & $\%$ & & \\
\hline Bactrocera mesomelas (Bezzi, 1908) & 1 & 0.03 & 1 & 0.00 & 0 & 0.00 & 1 & 0.15 & 3 & 0.00 \\
\hline Zeugodacus cucurbitae (Coquillet, 1899) & 1,401 & 38.69 & 4 & 0.01 & 19 & 1.00 & 0 & 0.00 & 1,424 & 2.07 \\
\hline Bactrocera dorsalis (Hendel, 1912) & 7 & 0.19 & 62,289 & 99.60 & 48 & 2.53 & 5 & 0.75 & 62,349 & 90.72 \\
\hline Ceratitis anonae (Graham, 1908) & 1 & 0.03 & 0 & 0.00 & 8 & 0.42 & 1 & 0.15 & 10 & 0.01 \\
\hline Ceratitis cosyra (Walker, 1849) & 29 & 0.80 & 13 & 0.02 & 1,038 & 54.63 & 9 & 1.35 & 1,089 & 1.58 \\
\hline Ceratitis ditissima (Munro, 1938) & 1 & 0.03 & 1 & 0.00 & 0 & 0.00 & 0 & 0.00 & 2 & 0.00 \\
\hline Ceratitis fasciventris (Bezzi, 1920) & 23 & 0.64 & 28 & 0.04 & 647 & 34.05 & 519 & 77.58 & 1,217 & 1.77 \\
\hline Ceratitis rubivora (Coquillet, 1901) & 4 & 0.11 & 0. & 0.00 & 0 & 0.00 & 6 & 0.90 & 10 & 0.01 \\
\hline Dacus bivittatus (Bigot, 1858) & 1,820 & 50.26 & 71 & 0.11 & 74 & 3.89 & 2 & 0.30 & 1,967 & 2.86 \\
\hline Dacus cf. eminus (Munro, 1939) & 1 & 0.03 & 0 & 0.00 & 0 & 0.00 & 0 & 0.00 & 1 & 0.00 \\
\hline Dacus humeralis (Bezzi, 1915) & 2 & 0.06 & 1 & 0.00 & 0 & 0.00 & 0 & 0.00 & 3 & 0.00 \\
\hline Dacus masaicus (Munro, 1937) & 10 & 0.28 & 0 & 0.00 & 0 & 0.00 & 0 & 0.00 & 10 & 0.01 \\
\hline Dacus punctatifrons (Karsh, 1887) & 256 & 7.07 & 19 & 0.03 & 12 & 0.63 & 0 & 0.00 & 287 & 0.42 \\
\hline Dacus telfaireae (Bezzi, 1924) & 30 & 0.83 & 0 & 0.00 & 5 & 0.26 & 0 & 0.00 & 35 & 0.05 \\
\hline Perilampsis pulchella (Austen, 1910) & 2 & 0.06 & 75 & 0.12 & 0 & 0.00 & 0 & 0.00 & 77 & 0.11 \\
\hline Ptiloniola edwardsi (Munro, 1967) & 24 & 0.66 & 12 & 0.02 & 1 & 0.05 & 1 & 0.15 & 38 & 0.06 \\
\hline Trirhithrum nigerrimum (Bezzi, 1913) & 9 & 0.25 & 22 & 0.04 & 16 & 0.84 & 4 & 0.60 & 51 & 0.07 \\
\hline Ceratitis lineata (Hering, 1938) & 0 & 0.00 & 1 & 0.00 & 0 & 0.00 & 0 & 0.00 & 1 & 0.00 \\
\hline Ceratitis capitata (Wiedemann, 1824) & 0 & 0.00 & 1 & 0.00 & 14 & 0.74 & 118 & 17.64 & 133 & 0.19 \\
\hline Bistrispinaria magniceps (Bezzi, 1824) & 0 & 0.00 & 0 & 0.00 & 1 & 0.05 & 0 & 0.00 & 1 & 0.00 \\
\hline $\begin{array}{l}\text { Ceratitis pallidula (De Meyer, Mwatawala } \\
\text { \& Virgilio, 2016) }\end{array}$ & 0 & 0.00 & 0 & 0.00 & 17 & 0.89 & 0 & 0.00 & 17 & 0.02 \\
\hline Ceratitis punctata (Wiedemann, 1824) & 0 & 0.00 & 0 & 0.00 & 0 & 0.00 & 1 & 0.15 & 1 & 0.00 \\
\hline Trirhithrum coffeae (Bezzi, 1918) & 0 & 0.00 & 0 & 0.00 & 0 & 0.00 & 2 & 0.30 & 2 & 0.00 \\
\hline Total & 3,621 & 100 & 62,538 & 100 & 1,900 & 100 & 669 & 100 & 68,728 & 100 \\
\hline$\%$ & & 5.27 & & 90.99 & & 2.76 & & 0.97 & & 100 \\
\hline Number of species & 17 & & 14 & & 13 & & 12 & & & \\
\hline
\end{tabular}

ME: methyl eugenol; CUE: cuelure; TA: terpinyl acetate; TRI: trimedlure. 
Table 5 Distribution of species according to localities.

\begin{tabular}{|c|c|c|c|c|c|c|c|c|c|c|c|c|c|c|c|}
\hline \multirow{3}{*}{$\begin{array}{l}\text { Species } \\
\text { Bactrocera mesomelas (Bezzi, 1908) }\end{array}$} & \multicolumn{14}{|c|}{ Distribution of species in localities } & \multirow{3}{*}{$\begin{array}{l}\text { Average } \\
\text { of } \% \\
0.05\end{array}$} \\
\hline & \multirow{2}{*}{$\begin{array}{l}\text { Cibitoke } \\
2\end{array}$} & \multirow{2}{*}{$\begin{array}{l}\% \\
0.30\end{array}$} & \multicolumn{2}{|c|}{ Bubanza \% } & \multicolumn{2}{|c|}{ Kigwena \% } & \multicolumn{2}{|c|}{ Kayanza \% } & \multirow{2}{*}{ Mwaro } & \multirow{2}{*}{$\begin{array}{l}\% \\
0.00\end{array}$} & \multirow{2}{*}{$\begin{array}{l}\text { Rumonge } \\
0\end{array}$} & \multirow{2}{*}{$\begin{array}{l}\% \\
0.00\end{array}$} & \multirow{2}{*}{$\begin{array}{l}\text { Bujumbura } \\
0\end{array}$} & \multirow{2}{*}{$\begin{array}{l}\% \\
0.00\end{array}$} & \\
\hline & & & 1 & 0.04 & 0 & 0.00 & 0 & 0.00 & & & & & & & \\
\hline Zeugodacus cucurbitae (Coquillet, 1899) & 27 & 3.99 & 277 & 12.42 & 3 & 0.08 & 0 & 0.00 & 0 & 0.00 & 21 & 1.16 & 1,096 & 1.89 & 2.79 \\
\hline Bactrocera dorsalis (Hendel, 1912) & 413 & 61.09 & 709 & 31.79 & 3,582 & 98.38 & 3 & 2.52 & 64 & 2.91 & 1,724 & 95.35 & 55,854 & 96.21 & 55.46 \\
\hline Bistrispinaria magniceps (Bezzi, 1918) & 0 & 0.00 & 1 & 0.04 & 0 & 0.00 & 0 & 0.00 & 0 & 0.00 & 0 & 0.00 & 0 & 0.00 & 0.01 \\
\hline Ceratitis anonae (Graham, 1908) & 0 & 0.00 & 1 & 0.04 & 1 & 0.03 & 0 & 0.00 & 7 & 0.32 & 0 & 0.00 & 1 & 0.00 & 0.06 \\
\hline Ceratitis capitata (Wiedemann, 1824) & 62 & 9.17 & 47 & 2.11 & 5 & 0.14 & 2 & 1.68 & 0 & 0.00 & 0 & 0.00 & 17 & 0.03 & 1.88 \\
\hline Ceratitis fasciventris (Bezzi, 1920) & 4 & 0.59 & 13 & 0.58 & 0 & 0.00 & 0 & 0.00 & 1,196 & 54.36 & 0 & 0.00 & 4 & 0.01 & 7.93 \\
\hline Dacus bivittatus (Bigot, 1858) & 63 & 9.32 & 1,075 & 48.21 & 0 & 0.00 & 32 & 26.89 & 537 & 24.41 & 60 & 3.32 & 200 & 0.34 & 16.07 \\
\hline Dacus cf. eminus (Munro, 1939) & 0 & 0.00 & 1 & 0.04 & 0 & 0.00 & 0 & 0.00 & 0 & 0.00 & 0 & 0.00 & 0 & 0.00 & 0.01 \\
\hline Dacus punctatifrons (Karsh, 1887) & 71 & 10.50 & 79 & 3.54 & 4 & 0.11 & 0 & 0.00 & 113 & 5.14 & 0 & 0.00 & 20 & 0.03 & 2.76 \\
\hline Trirhithrum nigerrimum (Bezzi, 1913) & 3 & 0.44 & 26 & 1.17 & 0 & 0.00 & 0 & 0.00 & 21 & 0.95 & 0 & 0.00 & 1 & 0.00 & 0.37 \\
\hline $\begin{array}{l}\text { Ceratitis pallidula (De Meyer, } \\
\text { Mwatawala \& Virgilio, 2016) }\end{array}$ & 0 & 0.00 & 0 & 0.00 & 17 & 0.47 & 0 & 0.00 & 0 & 0.00 & 0 & 0.00 & 0 & 0.00 & 0.07 \\
\hline Ceratitis cosyra (Walker, 1849) & 29 & 4.29 & 0 & 0.00 & 29 & 0.80 & 0 & 0.00 & 177 & 8.05 & 0 & 0.00 & 854 & 1.47 & 2.09 \\
\hline Ceratitis ditissima (Munro, 1938) & 1 & 0.15 & 0 & 0.00 & 0 & 0.00 & 0 & 0.00 & 0 & 0.00 & 0 & 0.00 & 0 & 0.00 & 0.02 \\
\hline Dacus humeralis (Bezzi, 1915) & 1 & 0.15 & 0 & 0.00 & 0 & 0.00 & 2 & 1.68 & 0 & 0.00 & 0 & 0.00 & 0 & 0.00 & 0.26 \\
\hline Ceratitis rubivora (Coquillet, 1901) & 0 & 0.00 & 0 & 0.00 & 0 & 0.00 & 10 & 8.40 & 0 & 0.00 & 0 & 0.00 & 0 & 0.00 & 1.20 \\
\hline Dacus masaicus (Munro, 1937) & 0 & 0.00 & 0 & 0.00 & 0 & 0.00 & 10 & 8.40 & 0 & 0.00 & 0 & 0.00 & 0 & 0.00 & 1.20 \\
\hline Dacus telfaireae (Bezzi, 1924) & 0 & 0.00 & 0 & 0.00 & 0 & 0.00 & 4 & 3.36 & 31 & 1.41 & 0 & 0.00 & 0 & 0.00 & 0.68 \\
\hline Perilampsis pulchella (Austen, 1910) & 0 & 0.00 & 0 & 0.00 & 0 & 0.00 & 16 & 13.45 & 54 & 2.45 & 0 & 0.00 & 7 & 0.01 & 2.27 \\
\hline Ptiloniola edwardsi (Munro, 1967) & 0 & 0.00 & 0 & 0.00 & 0 & 0.00 & 38 & 31.93 & 0 & 0.00 & 0 & 0.00 & 0 & 0.00 & 4.56 \\
\hline Trirhithrum coffeae (Bezzi, 1918) & 0 & 0.00 & 0 & 0.00 & 0 & 0.00 & 2 & 1.68 & 0 & 0.00 & 0 & 0.00 & 0 & 0.00 & 0.24 \\
\hline Ceratitis ditissima (Munro, 1938) & 0 & 0.00 & 0 & 0.00 & 0 & 0.00 & 0 & 0.00 & 0 & 0.00 & 1 & 0.06 & 0 & 0.00 & 0.01 \\
\hline Ceratitis lineata (Hering, 1938) & 0 & 0.00 & 0 & 0.00 & 0 & 0.00 & 0 & 0.00 & 0 & 0.00 & 1 & 0.06 & 0 & 0.00 & 0.01 \\
\hline Ceratitis punctata (Wiedemann, 1824) & 0 & 0.00 & 0 & 0.00 & 0 & 0.00 & 0 & 0.00 & 0 & 0.00 & 1 & 0.06 & 0 & 0.00 & 0.01 \\
\hline Total & 676 & 100 & 2,230 & 100 & 3,641 & 100 & 119 & 100 & 2,200 & 100 & 1,808 & 100 & 58,054 & 100 & 100 \\
\hline Number of species & 11 & & 11 & & 7 & & 10 & & 9 & & 6 & & 10 & & \\
\hline
\end{tabular}


Table 6 Similarity indices of Sorensen (\%) between the different localities.

\begin{tabular}{|c|c|c|c|c|c|c|}
\hline Localities & Bubanza & Mwaro & Rumonge & Kigwena & Bujumbura & Cibitoke \\
\hline \multicolumn{7}{|l|}{ Bubanza } \\
\hline Mwaro & 60 & & & & & \\
\hline Rumonge & 37.5 & 26.666 & & & & \\
\hline Kigwena & 55.555 & 50 & 30.769 & & & \\
\hline Bujumbura & 76.190 & 84.210 & 37.5 & 66.666 & & \\
\hline Cibitoke & 76.190 & 60 & 35.294 & 55.555 & 76.190 & \\
\hline Kayanza & 28.571 & 44.444 & 25 & 23.529 & 40 & 30.095 \\
\hline
\end{tabular}

showing that the distribution of $D$. bivittatus was limited to sites above an altitude of $1,640 \mathrm{~m}$. C. fasciventris was the most abundant species at Mwaro (54.36\%). Species present at Mwaro $(1,640 \mathrm{~m})$ showed higher abundance in the other localities.

The average percentage of the seven localities shows that $B$. dorsalis was most abundant in Burundi with an average of $55.46 \%$, followed by $D$. bivittatus at $16.07 \%$ and C. fasciventris (7.93\%). The species $P$. edwardsi made up $4.56 \%$ of trap captures in Burundi and was concentrated at only one locality (Kayanza). Other species are poorly represented at this site. This could be due to altitude or ecosystem.

\subsection{Similarity between Localities}

The index of Sorensen found is more than $50 \%$ for some localities, whereas for others it is less than $50 \%$ (Table 6).

The index of Sorensen indicated the highest similarity between localities Mwaro and Bujumbura (84.120\%), Cibitoke and Bujumbura (76.190\%), Cibitoke and Bubanza (76.190\%), Bubanza and Bujumbura (76.190\%).

A medial similarity existed between Bujumbura and Kigwena (66.666\%), Mwaro and Bubanza (60\%), Mwaro and Cibitoke (60\%), Kigwena and Bubanza and Cibitoke and Kigwena (55.555\%).

The lowest similarity was between localities Kigwena and Kayanza (23.529\%), Rumonge and Kayanza (25\%), Kayanza and Bubanza (28.571\%).

According to these values abovementioned, it can be concluded that the similarity between localities doesn't depend on altitude.

\section{Discussion}

\subsection{Fruit Flies and Non-fruit Flies Captured}

The number of fruit flies captured are far superior to those of non-fruit flies. This would be due to the fact that parapheromones are products intended to attract species of fruit flies [18]. Nevertheless, species of non-fruit flies captured can not be considered as improvised species, given that they have been caught in research done elsewhere. It could also been said that the presence of non-fruit flies in the traps may be due to accidental introductions.

The results show that the parapheromone products used in this study attract Tephritidae species at far greater strength than other insects. Nevertheless, the capture of non-target species could not be considered as negligible, given that they have been recorded in other studies. In Hawaii, flower-visiting insects, such as bees [19], Anthidium sp. [20] and syrphid flies Melanostoma mellinum, Meliscaeva cincetella, Blera scitula, Hadromyia pulcha [21] have been recorded in small quantities by methyl eugenol containing traps. Drosphilidae was also found in the traps in this study; this may be due to dead flies in the traps [19]. Uchida et al. [22] found that there was a relationship between the parapheromone used and the non-target species captured. In this study it was found that methyl eugenol had a high incidence of non-target species. This supports results found by Leblanc et al. [23] with regards to Diptera and Hymenoptera. In Ghana, Foba et al. [24] found that methyl eugenol and trimedlure captured non-target species from eight different insect orders and one arachnid (Arachnida). Due to the low 
numbers of non-target species in the traps, it can be concluded that these captures are coincidental. The non-fruit flies are also either attracted to moisture in the traps or the yellow color of the trap.

\subsection{Species Caught by Parapheromones}

In this study, 24 species of fruit flies were recorded in Burundi. However, this list is only representative of a selected area, where genera Bactrocera, Dacus, Bistrispinaria, Trirhithrum, Perilampsis, Ceratitis and Zeugodacus were recorded. The research has highlighted the presence of two exotic species, $B$. dorsalis and Z. cucurbitae. These species have a wide geographical distribution, as recorded by French [25], Drew et al. [26] and Virgilio et al. [27]. These two species are severe threat to the agricultural sector of Burundi.

Five species recorded from this study, including the two exotic invasive species, have been recorded in neighboring countries, such as Democratic Republic of Congo [28, 29], Rwanda [30] and Tanzania [31]. These species are $B$. dorsalis, D. bivittatus, D. punctatifrons, D. humeralis Bezzi and C. fasciventris. $B$. dorsalis has greatly effect on the agricultural sector of the Great Lakes region (Burundi, Democratic Republic of Congo, Rwanda and Uganda). Effective control methods for this species are needed throughout this region, such as those initiated in Tanzania. Without an area wide control, neighboring populations will continuously re-infest these countries.

It was shown in this study that $C$. anonae Graham, C. capitata, C. cosyra and C. punctata Wiedemann are present in Burundi, and they are also present in Democratic Republic of Congo [29]. C. capitata, C. cosyra, C. punctata and C. rubivora are present in Burundi and Tanzania. C. fasciventris is present in Rwanda.

The species Z. cucurbitae is present in Burundi and Tanzania [32]. This species is absent in Rwanda and the Democratic Republic of Congo. The species Trirhithrum nigerrimum Bezzi detected in this study is also present in the Democratic Republic of Congo [28], while the species T. coffeae Bezzi is also present in Tanzania [31]. It is noted that the species $D$. masaicus Munro, C. ditissima Munro, Bistrispinaria magniceps Bezzi, B. mesomelas Bezzi, Ptioniola edwardsi and D. cf. eminus Munro are present in Burundi, but not in the neighboring countries [29-31].

\subsection{Effect of Parapheromones}

Bactrocera, Perilampsis and Trirhithrum were extensively captured by methyl eugenol. Other species of the genera Ceratitis, Dacus, Zeugodacus and Ptioniola were attracted to a lesser extent by methyl eugenol. However, C. rubivora, C. anonae, C. punctata, D. masaicus, D. telfaireae Bezzi, D. cf. eminus and $T$. coffeae were not attracted. These results coincide with those of Mwatawala et al. [31] and Alvin et al. [33], showing the sensitivity of B. dorsalis to methyl eugenol.

Cuelure captured Z. cucurbitae, D. bivittatus and D. punctatifrons extensively. In addition to these three species, cuelure captured other species of the genera Ceratitis, Trirhithrum and Bactrocera to a lesser extent. Vayssières et al. [34], De Meyer et al. [35] and Dhillon et al. [36] have found the same results for $Z$. cucurbitae, D. bivittatus and D. punctatifrons. The capture of $B$. dorsalis by cuelure is an abnormal event, as males of economically important Tephritidae may be attracted to either cuelure or methyl eugenol, but never to both [37]. This recording may be due to possible contamination or coincidental attraction to the yellow trap.

Terpinyl acetate captured C. fasciventris and $C$. cosyra in abundance and C. anonae to a lesser extent. These results are in accordance with those found by Vayssières et al. [34]. Terpinyl acetate is known to attract the species of the genus Ceratitis [3], but in this study, attraction of species from the genera Dacus, Trirhithrum, Ptioniola and Bactrocera was recorded. The same phenomenon is observed in trimedlure, which was developed as a lure for C. capitata [16]. 


\subsection{Distribution of Species by Localities}

Taking into account all species captured, B. dorsalis was the most abundant and present in all the localities inspected. However, its abundance differed from one locality to another, confirming its invasive nature [26]. Its abundance was the highest in the localities of low altitude, especially in Bujumbura. The abundance decreased with an increase in altitude with the lowest abundance at Kayanza located at high altitude.

C. fasciventris seems to be a species of high altitude, as it was most abundant at an altitude of $1,640 \mathrm{~m}$ (Mwaro) than at an altitude of 1,101 m (Bubanza). $C$. anonae was found throughout low altitude, on average elevation and high altitude. These two species are present throughout East Africa [38, 39].

C. cosyra was abundant at low altitude at Bujumbura. Although it was abundant in Bujumbura, it was dominated by $B$. dorsalis in terms of numbers. Mwatawala et al. [8] and Ekesi et al. [40] found that the presence of $B$. dorsalis lead to a decrease in the abundance of C. cosyra. D. bivittattus was abundant at medium altitude, while $D$. punctatifrons was abundant at medium and high altitudes. The other species caught were present at low numbers throughout and had no correlation to altitude.

\subsection{Similarity between Localities}

The index of Sorensen shows in general that some localities had the same species, but the altitudinal configuration was not confirmed. The localities of Mwaro and Bujumbura with a high similarity $(84.120 \%)$ were a low altitude and high altitude sight, respectively. On the contrary, the localities of Cibitoke and Kigwena had a low similarity (55.555\%) and both were located at low altitude. The similarity is not linked to the altitude rather to the presence of a particular species at a given locality. This presence or absence of a species is due to biotic or abiotic factors [41-43]. The great similarity observed between Bujumbura and Mwaro would be due in large part to biotic factors. Indeed, some fruit plants in the city of Bujumbura are also found at Mwaro. Biotic factors contribute in the sustainability of the populations of fruit flies in these two localities.

\section{Conclusions}

Burundi is a country rich in species of Tephritid fruit flies. In addition to the species specific to the African continent, there are other exotic species. $B$. dorsalis and Z. cucurbitae are a great threat to the horticultural sector of Burundi. These results demonstrated the need for an effective control strategy to lessen the damage caused by these dreaded pests. Results showed that methyl eugenol was a highly effective lure for $B$. dorsalis and further research efforts should focus on using this in control programs.

\section{Acknowledgments}

This publication is the result of combined efforts of several stakeholders. Sincere thanks go to the General Director of OBPE for granting for the export of the samples to be identified. Special thanks to the RMCA for the fellowships awarded and the mentoring of students during their internships. Also gratitude is expressed to Dr. Marc De Meyer, specialist in entomology at RMCA for giving help both in the identification and confirmation of the frugivorous fruit fly species, as well as the correction of this article before its publication. Many thanks go to Nyabenda Mathias, technician in the Laboratory of Biodiversity at OBPE, as well as to all the students who participated in this research.

\section{References}

[1] Salum, J. K., Mwatawala, M. W., Kusolwa, P. M., and De Meyer, M. 2013. "Demographic Parameters of the Two Main Fruit Fly (Diptera: Tephritidae) Species Attacking Mango in Central Tanzania." J. Appl. Entomol. 138 (6): 441-8.

[2] Lux, S. A. 1999. African Fruit Fly Initiative: Pan-African Initiative to Promote Productivity and Trade of Fruits and Vegetables through Management of African Fruit Flies. International Centre for Insect Physiology and 
Ecology (ICIPE) Project Proposal, Nairobi.

[3] IAEA. 2003. Trapping Guidelines for Area-Wide Fruit Fly Programmes.

[4] Lux, S. A., Ekesi, S., Dimbi, S., Mohamed, S., and Billah, M. 2003. "Mango-Infesting Fruit Flies in Africa: Perspectives and Limitations of Biological Approaches to Their Management." In Biological Control in IPM Systems in Africa, edited by Neuenschwander, P., Borgemeister, C., and Langewald, J. Wallingford: CABI Publishing, 277-94.

[5] Mwatawala, M. W., Mziray, H., Malebo, H., and De Meyer, M. 2015. "Guiding Farmers' Choice for an Integrated Pest Management Program against the Invasive Bactrocera dorsalis Hendel (Diptera: Tephritidae) in Mango Orchards in Tanzania." Crop Protection 76: 103-7.

[6] White, I. M., and Elson-Harris, M. M. 1992. Fruit Flies of Economic Significance: Their Identification and Bionomics. Wallingford, UK: CAB International.

[7] Kibira, M., Affognon, H., Njehia, B., Muriithi, B., and Ekesi, S. 2015. "Economic Evaluation of Integrated Management of Fruit Fly in Mango Production in Embu County, Kenya." Afr. J. Agric. Resour. Econ. 10 (4): 343-53.

[8] Mwatawala, M. W., De Meyer, M., Makundi, R. H., and Maerere, A. P. 2009. "Host Range and Distribution of Fruit-Infesting Pestiferous Fruit Flies (Diptera, Tephritidae) in Selected Areas of Central Tanzania." Bull. Entomol. Res. 99 (6): 629-41.

[9] Mwatawala, M. W., De Meyer, M., Makundi, R. H., and Maerere, A. P. 2006. "Seasonality and Host Utilization of the Invasive Fruit Fly, Bactrocera invadens (Dipt., Tephritidae) in Central Tanzania." Journal of Applied Entomology 130 (9-10): 530-7.

[10] Project to Support Economic Management (PAGE). 2007. Integrator Report Supporting the Diagnosis of the Value Chain and the Development of the Strategy of the Horticulture of Burundi. (in French)

[11] Ministry of Agriculture and Livestock (MINAGRI). 2013. Strategic Framework for the Development of Horticulture in Burundi. (in French)

[12] Mwatawala, M. W., De Meyer, M., Makundi, R. H., and Maerere, A. P. 2009. "Design of an Ecologically Based IPM Program for Fruit Flies (Diptera: Tephritidae) in Tanzania." Fruits 68: 83-90.

[13] Modjonnesso, G., Komina, A., Yaovi, N., Banibéa, S. B., Essè, A. K., and Glitho, A. I. 2012. "Species Diversity of Fruit Flies (Diptera: Tephritidae) in Two Ecological Zones of Togo." European Journal of Scientific Research 72 (3): 423-39.

[14] Vargas, R. I., Haviland, D., Faber, B., Kabashima, J., Grafton-Cardwell, B., and Morse, J. G. 2013. "Improving
Trapping Systems for Early Detection and Eradication of Fruit Flies in California." Citrograph 4 (4): 28-34.

[15] Leraut, P. 2003. The Entomological Guide, 527. (in French)

[16] Ekesi, S., and Billah, M. K. 2007. A Field Guide to the Management of Economically Important Tephritid Fruit Flies in Africa. Nairobi, Kenya: ICIPE Science Press, 96.

[17] Sorensen, T. 1948. "A Method of Establishing Groups of Equal Amplitude in Plant Ecology Based on Similarity of Species Content and Its Application to Analyses of the Vegetation on Danish Commons.” K. Dan. Vidensk. Selsk. Biol. Skr. 5: 1-34.

[18] Siivinski, J. M., and Carrol, C. 1986. "Pheromones and Parapheromones in the Control of Tephritids." The Florida Entomologist 69 (1): 157-68.

[19] Vargas, R. I., Shelly, T. E., Leblanc, L., and Piñero, J. C. 2010. "Recent Advances in Methyl Eugenol and Cuelure Technologies for Fruit Fly Detection, Monitoring and Control in Hawaii." Vitamins and Hormones 83: 575-95.

[20] Ndayizeye, L. 2016. Fruit Flies in the Western Region of Burundi. Saarbrücken, Germany: Lambert Academic Publishing (LAP), 74.

[21] Dowell, R. V. 2015. "Attraction of Non-target Insects to Three Male Fruit Fly Lures in California." Pan-Pacific Entomologist 91 (1): 1-19.

[22] Uchida, G. K., Mackey, B. E., McInnis, D. O., and Vargas, R. I. 2007. "Attraction of Bactrocera dorsalis (Diptera: Tephritidae) and Non-target Insects to Methyl Eugenol Bucket Traps with Different Preservative Fluids on Oahu Island, Hawaiian Islands." J. Econ. Entomol. 100 (3): 723-9.

[23] Leblanc, L., Vargas, R. I., and Rubinoff, D. 2010 "Captures of Pest Fruit Flies (Diptera: Tephritidae) and Non-target Insects in BioLure and Torula Yeast Traps in Hawaii." Environ. Entomol. 39 (5): 1626-30.

[24] Foba, C. N., Afreh-Nuamah, K., Billah, M. K., and Obeng-Ofori, D. 2012. "Species Composition of Fruit Flies (Diptera: Tephritidae) in the Citrus Museum at the Agricultural Research Centre (ARC), Kade, Ghana." Int. J. Tropic. Insect Sci. 32 (1): 12-23.

[25] French, C. 2005. "The New Invasive Bactrocera Species." In Insect Pest Control Newsletter No. 65. Vienna, Austria: International Atomic Energy Agency, 19-20.

[26] Drew, R. A. I., Tsuruta, T., and White, I. M. 2005. “A New Species of Pest Fruit Fly (Diptera: Tephritidae: Dacinae) from Sri Lanka and Africa." Afr. Entomol. 13 (1): 149-54.

[27] Virgilio, M., Delatte, H., Backeljau, T., and De Meyer, M. 2010. "Macrogeographic Population Structuring in the Cosmopolitan Agricultural Pest Bactrocera cucurbitae (Diptera: Tephritidae)." Molecular Ecology 19 (13): 2713-24. 
[28] Virgilio, M., Backeljau, T., Emeleme, R., and Juakali, J. L. 2011. "A Quantitative Comparison of Frugivorous Tephritids (Diptera: Tephritidae) in Tropical Forests and Rural Areas of the Democratic Republic of Congo." Bulletin of Entomological Research 101 (5): 591-7.

[29] Rubabura, K., Munyuli, B. M. T., Bisimwa, B. E., and Kazi, K. S. 2015. "Invasive Fruit Fly, Ceratitis Species (Diptera: Tephritidae), Pests in South Kivu Region, Eastern of Democratic Republic of Congo.” Int. J. Innov. Sci. Res. 16 (2): 403-8.

[30] Harelimana, A., Le Goff, G., Rukazambuga, D., Ngamata, M. O., and Hance, T. 2016. "Assessment of Fruit Fly Bactrocera invadens (Diptera: Tephritidae) Presence in Eastern Province of Rwanda." East African Journal of Science and Technology 6 (2): 1-9.

[31] Mwatawala, M. W., De Meyer, M., Makundi, R. H., and Maerere, A. P. 2006. "Biodiversity of Fruit Flies (Diptera: Tephritidae) at Orchards in Different Agro-ecological Zones of the Morogoro Region, Tanzania.” Fruits 61 (5): 321-32.

[32] Mwatawala, M., Maerere, A. P., Makundi, R., and De Meyer, M. 2010. "Incidence and Host Range of the Melon Fruit Fly Bactrocera cucurbitae (Coquillett) (Diptera: Tephritidae) in Central Tanzania.” Int. J. Pest Manag. 56 (3): 265-73.

[33] Alvin, K. W. H., Ooi, Y. S., Wee, S. L., and Tan, K. H. 2015. "Comparative Sensitivity to Methyl Eugenol of Four Putative Bactrocera dorsalis Complex Sibling Species-Further Evidence That They Belong to One and the Same Species B. dorsalis." Zookeys 540: 313-21.

[34] Vayssières, J. F., Adandonon, A., Sinzogan, A., and Korie, S. 2010. "Diversity of Fruit Fly Species (Diptera: Tephritidae) Associated with Citrus Crops (Rutaceae) in Southern Benin in 2008-2009.” Int. J. Biol. Chem. Sci. 4 (6): 1881-97.

[35] De Meyer, M., Quilici, S., Franck, A., Chadhouliati, A.
C., Issimaila, M. A., Youssoufa, M. A., Abdoul-Karime, A. L., Barbet, A., Attié, M., and White, I. M. 2012. "Records of Frugivorous Fruit Flies (Diptera: Tephritidae: Dacini) from the Comoro Archipelago." African Invertebrates 53 (1): 69-77.

[36] Dhillon, M. K., Singh, R., Naresh, J. S., and Sharma, H. C. 2005. "The Melon Fruit Fly, Bactrocera cucurbitae: A Review of Its Biology and Management.” J. Insect Sci. 5: 40.

[37] Hardy, D. E. 1979. "Review of Economic Fruit Flies of the South Pacific Region." Pacific Insects 20: 429-32.

[38] Barr, N. B., and McPheron, B. A. 2006. "Molecular Phylogenetics of the Genus Ceratitis (Diptera: Tephritidae)." Mol. Phylogenet. Evol. 38 (1): 216-30.

[39] Copeland, R. S., Wharton, R. A., Luke, Q., De Meyer, M., Lux, S., Zenz, N., Machera, P., Okumu, M. 2006. "Geographic Distribution, Host Fruit and Parasitoids of African Fruit Fly Pests Ceratitis anonae, Ceratitis cosyra, Ceratitis fasciventris and Ceratitis rosa (Diptera: Tephritidae) in Kenya." Annals of the Entomological Society of America 99 (2): 261-78.

[40] Ekesi, S., Billah, M. K., Nderitu, P. W., Lux, S. A., and Rwomushana, I. 2009. "Evidence for Competitive Displacement of Ceratitis cosyra by the Invasive Fruit Fly Bactrocera invadens (Diptera: Tephritidae) on Mango and Mechanisms Contributing to the Displacement." $J$. Econ. Entomol. 102 (3): 981-91.

[41] Canadas, Á., Rade, D., and Zambrano, C. 2014. "Diptera (Tephritidae) and Their Relation with Biotic Factors in Santa Elena Region, Ecuador.” Rev. Colomb. Entomol. 40 (1): 55-62.

[42] Ganie, S. A., Khan, Z. H., Ahangar, R. A., Bhat, H. A., and Hussain, B. 2013. "Population Dynamics, Distribution and Species Diversity of Fruit Flies on Cucurbits in Kashmir Valley, India.” J. Insect Sci.13: 65.

[43] Kiritani, K. 1998. "Exotic Insects in Japan." Entomol. Sci. 1: $291-8$ 